\title{
O Programa Bolsa Família e a Oferta de Trabalho: Evidências para o Brasil e Paraná
}

\author{
Dryelli Jales Costa ${ }^{1}$ \\ Marina Silva da Cunha
}

\begin{abstract}
Resumo: O Programa Bolsa Família (PBF) afirmou-se no decorrer de uma década como um dos principais programas de transferência de renda condicionada no mundo, tendo como objetivo fundamental o fornecimento de condições para redução da pobreza e desigualdade de renda de curto e longo prazo. Todavia questionamentos quanto à eficácia do programa só podem ser solucionados com mecanismos pertinentes de avaliação. Para tal, utilizou-se amostras da PNAD 2006 para identificar os domicílios que foram beneficiados pelo PBF e também estabelecer diferenciações entre os gêneros de posições domiciliares diversificadas no Paraná e Brasil. Ao utilizar o método de Pareamento pelo Escore de Propensão, os resultados revelam que O PBF não reduz a participação no mercado de trabalho para as mulheres, e este efeito é mais predominante para as cônjuges. Desse modo, evidencia-se que o PBF não representa um fator de desestímulo ao trabalho, tendo em vista que a probabilidade de participação no mercado de trabalho não é reduzida para os beneficiários em grande parte dos subgrupos analisados.
\end{abstract}

Palavras chave: Programa Bolsa Família, mercado de trabalho, Pareamento pelo Escore de Propensão.

Classificação JEL: H54; R22; R53. 


\title{
The Bolsa Família Program and the labour supply: evidences for Brazil and Paraná
}

\begin{abstract}
The Bolsa Família Program (BFP) said in the course of a decade as one of the main programs of conditional cash transfers in the world, with the ultimate goal of providing conditions for reducing poverty and income inequality in the short and long term. However questions about the effectiveness of the program can only be solved with relevant evaluation mechanisms. To this end, we used samples from PNAD 2006 to identify households that benefited from the BFP and also establish differences between genders diversified household positions in $\mathrm{Pa}-$ rana and Brazil. By using the method of pairing the propensity score, the results reveal that the BFP does not reduce participation in the labor market for women, and this effect is most prevalent for spouses. Thus, it is evident that the BFP is not a discouraging factor to work, given that the probability of participation in the labor market is not reduced for beneficiaries in most subgroups analyzed.
\end{abstract}

Keywords: Bolsa Família Program; labor market; Propensity Score Matching.

JEL Classification: H54; R22; R53.

\section{Introdução}

As políticas públicas de caráter assistencialista no Brasil e na maioria dos países da América Latina seguiram uma tendência de maior focalização durante a última década. Objetivando alcançar melhorias significativas na qualidade da aplicação dos recursos o Governo Federal passou a investir, principalmente a partir dos anos 2000, em ações formais mais sólidas de identificação e classificação das famílias com perfil necessário para ingresso em seus programas de políticas públicas, sendo que o maior expoente atualmente é o Programa Bolsa Família (PBF) de 2003.

Apesar dos níveis de desigualdade ainda estarem em patamares elevados, nos últimos anos eles têm sofrido uma queda expressiva e contínua. Grande parte desse declínio pode ser explicado pelas transferências governamentais, como apontado por Barros et al. (2007). Segundo o autor, o PBF teve uma participação correspondente a $12 \%$ na queda da desigualdade medida pelo Índice de Gini no período de 2001 à 2005.

Estudos a respeito dos programas de transferência de renda condicionada ajudam a interpretar os resultados dos incentivos e desestímulos desencadeados com o seu recebimento, especialmente quanto ao mercado laboral. 
O primeiro problema encontrado em estudos empíricos desse tipo é sobre a queda voluntária no número de horas trabalhadas para que a renda atinja o critério de elegibilidade por parte dos indivíduos, como tratado por Bichir (2010). Já a segunda problemática deve-se à presença de efeitos diferenciados entre os membros que recebem o benefício e suas posições no grupo familiar. De acordo com Teixeira (2010), a exigência de que os filhos entre 6 e 15 anos tenham frequência escolar pode fazer com que horas a mais sejam liberadas para maior participação no mercado de trabalho por parte dos pais ou responsáveis.

A averiguação a respeito dos efeitos do PBF na participação do mercado de trabalho no Brasil e Paraná é o foco principal desse estudo. Para maior aprofundamento partiu-se da suposição de que rendas desvinculadas ao trabalho, como as provenientes dos programas assistencialistas, podem gerar efeitos na função de produção domiciliar, além de representar um choque na renda interna da família. Assim, tendo em vista a amplitude dos choques orçamentários, cabe esclarecer questões quanto ao recebimento do PBF e se este pode influenciar na elevação da participação dos membros do domicílio no mercado de trabalho.

Para avaliar os objetivos será utilizado como metodologia o Pareamento por Escore de Propensão, que identifica os grupos de beneficiários e não beneficiários com características mais próximas um do outro. Este trabalho se divide em sete sessões. A primeiramente a introdução, seguida da primeira sessão que trata sobre a Teoria da alocação do tempo, renda e estrutura familiar. A segunda sessão fala sobre o Programa Bolsa Família (PBF) e a construção da base de dados. O tópico três expõe detalhes sobre o método de análise de causalidade, o tópico quatro trata sobre o pareamento pelo escore de propensão, o tópico cinco que evidência o efeito do PBF na participação de homens e mulheres no mercado de trabalho e a última sessão que traz as considerações finais.

\section{Teoria da alocação do tempo, renda e estrutura fami-

De acordo com Becker (1976) e Gronau (1986) o salário total, valor da hora de trabalho, renda desvinculada com o trabalho e a divisão intradomiciliar são fatores preponderantes para a decisão do tempo que será alocado com trabalho. Desse modo, alterações na composição da renda do domicílio podem modificar a quantidade de trabalho ofertada por seus membros. Aumentos na renda acarretam choques positivos para o domicílio, todavia o PBF não representa um choque de renda vinculado ao trabalho.

Partindo da teoria da alocação do tempo de Becker (1965) e da produção domiciliar de Becker (1976), é possível analisar a função de oferta de traba- 
lho individual. A teoria sugere que a decisão de alocação temporal envolve o tempo dedicado aos afazeres domésticos, tais como educação dos filhos e ao trabalho remunerado. Na função de produção domiciliar, o tempo dedicado aos afazeres domésticos torna-se uma variável importante para transformação de bens e serviços adquiridos em produtos prontos para o consumo. Este tempo colabora para o aumento do bem-estar, gerando utilidade.

Modificações na renda, como um choque provocado pelo PBF, gera novas alocações para o tempo entre afazeres domésticos e trabalho remunerado. Aumentos súbitos não provenientes do trabalho elevam o tempo despendido com afazeres domésticos comparado com o tempo gasto com trabalho remunerado. Assim, como discutido por Paker e Skoufias (2000), programas de transferência de renda podem gerar desincentivos ao trabalho remunerado (efeito-renda), já que pode criar paralelamente incentivos ao trabalho doméstico ou lazer.

Todavia, a uniformidade da sensibilidade ao choque orçamentário acarretado com as transferências monetárias entre os domicílios não é esperada, uma vez que cada membro toma suas decisões de alocação do tempo baseado normalmente nos demais membros.

A divisão do trabalho no domicílio implica também o compartilhamento de recursos e do tempo total disponível. Gronau (1977) cita dois modelos que ajudam a explicar o processo de tomada de decisão a respeito das escolhas de consumo e oferta de trabalho dos membros familiares, são eles: modelos unitário e coletivo. No primeiro, presume-se que a família maximiza sua utilidade conjuntamente, levando em conta a restrição orçamentária de todos os membros e descartando as preferências individuais. Já o segundo afirma que a interação das preferências distintas quanto ao consumo e lazer leva a um resultado eficiente no sentido de Pareto ${ }^{1}$.

Estudos a respeito do conceito de "efeito trabalhador adicional" na dinâmica familiar foram desenvolvidos por Duryea et al. (2003) e Stephens (2001). O conceito trata da substituição por parte dos membros do domicílio do tempo alocado em trabalho remunerado e em afazeres domésticos. Entretanto, o efeito do trabalhador adicional sustenta a ideia de que a substituição intradomiciliar obedece à uma "hierarquia" entre os membros da família, sendo que fatores como sexo, idade e papeis no domicílio ajudam a definir mais detalhadamente a dinâmica para faixas de renda diferenciadas.

Cardoso (1999), ao tratar do mercado de trabalho, ressaltou o fato da interação entre produção doméstica e oferta de trabalho se elevar à medida que o trabalho se torna menos formalizado. Isto ocorre principalmente quando há predominância do trabalho por conta própria, já que as atividades domésticas se confundem com a produção. Desse modo, não basta olhar a destinação do benefício exclusivamente para o consumo, que por si só já representa uma

1 Para maiores detalhes ver CHIAPPORI (1992). 
melhoria na qualidade de vida das famílias beneficiadas, outros destinos dados aos valores recebidos permitem pensar em impactos positivos na oferta de trabalho individual.

Programas de transferências de renda podem gerar efeitos diversificados à medida que aumentam a renda domiciliar e diminuem a renda individual, contudo apesar dessas hipóteses serem factíveis, a afirmação a respeito da participação no mercado de trabalho não pode ser conclusiva de acordo com Ehrenberg e Smith (2003), já que há possibilidade de substituição da produção doméstica pelos cônjuges, o que justifica o estudo dos efeitos do PBF sobre cada membro e sua posição no domicílio.

Ferrario e Cunha (2012) evidenciam o trabalho de Becker (1981) sobre o tratado da família, em que se observou que as estruturas familiares vêm se alterando, especialmente a partir da década de 1950. Becker (1981) aponta ainda, como exemplificação, o caso americano sobre crescimento dos divórcios e elevação das famílias chefiadas por mulheres e o número de crianças em famílias com um responsável apenas. A queda acentuada da taxa de natalidade também foi destacada como um dos pontos que mais colaborou na redução do tamanho das famílias e com a maior participação da mulher nas atividades econômicas.

Contudo, Brito (2008) salienta a disparidade do crescimento populacional entre os diferentes grupos sociais, devido a transição demográfica que vem ocorrendo desde a segunda metade do século, em que as taxas de fecundidade e mortalidade diminuíram. Desse modo, categorias de renda inferior têm probabilidade mais alta de terem nascido na primeira metade desse século. $\mathrm{O}$ autor ainda destaca o nível de fecundidade maior entre as mulheres com menor nível de renda per capita, assim, famílias mais numerosas e com mais filhos estão associadas na maioria das vezes a famílias mais pobres e consequentemente as que mais recorrerão aos programas assistenciais.

\section{O Programa Bolsa Família e a Construção da base de dados}

A partir da criação do PBF em 2003, possibilitaram-se duas evoluções significativas no sistema brasileiro de proteção social: o primeiro refere-se a programas similares já existentes e sua unificação, uma vez que a maioria operava separadamente, eliminando lacunas e ineficiências; já o segundo trata da elevação da cobertura social, com destaque para a população pobre em idade ativa, especificamente as crianças.

A partir da Pesquisa Nacional por Amostra de Domicílios (PNAD) do ano de 2006, ano adotado neste estudo, iniciou-se a construção da base de dados levando em conta os critérios de elegibilidade do programa. A escolha da 
pesquisa básica desse ano deu-se pelo fato da mesma vir acompanhada de um suplemento que mostra as características de acesso a transferências de renda de programas sociais ${ }^{2}$. Desse modo se permitiu a identificação de pelo menos um morador do domicílio que era beneficiário do PBF, utilizando a variável V2403 da PNAD.

Inicialmente, a estimativa para identificar a probabilidade de participação do PBF é realizada por dois grupos com distinção apenas do sexo dos moradores do domicílio. A análise é efetuada tanto para o Brasil quanto para o Estado do Paraná. Posteriormente é verificado mais especificamente dois grupos contendo o sexo e a posição familiar dos membros. O primeiro grupo é composto por mulheres e homens de referência ou chefes no domicílio; e o segundo grupo engloba as mulheres e homens cônjuges. É utilizado o programa STATA 11.2 para obter as estimações necessárias para as avaliações.

A amostra selecionada abrange domicílios com renda per capita compreendida entre $\mathrm{R} \$ 1,00$ e $\mathrm{R} \$ 200,00$. A utilização de um cut-off mais elevado do que os $\mathrm{R} \$ 120,00$ necessários para que se obedeça ao critério de elegibilidade do PBF se dá pela tentativa de inclusão de beneficiários que declararam no Cadastro Único uma renda mais baixa do que a que consta na PNAD, o que os torna elegíveis incorretamente. Desse modo o valor de $\mathrm{R} \$ 200,00$ foi escolhido aleatoriamente estando compreendido entre $\mathrm{R} \$ 260,00$, que corresponde ao valor do salário mínimo em setembro de 2006 e os $\mathrm{R} \$ 120,00$, fundamentais para obedecer aos critérios do programa.

TABELA 1: DESCRIÇÃO DAS VARIÁVEIS

\begin{tabular}{l|c}
\hline Variável & Descrição \\
\hline TREAT & $\begin{array}{c}\text { Recebe tratamento (PBF=1) e não recebe tratamento } \\
\text { PBF })\end{array}$ \\
$\begin{array}{l}\text { Participa } \\
\text { Idade }\end{array}$ & $\begin{array}{c}\text { Participa do mercado de trabalho } \\
\text { idade2 }\end{array}$ \\
Raça & Idade dos membros entre 15 e 65 anos \\
estudo1 & Idade ao quadrado \\
estudo2 & Raça do membro familiar (nbranca=0; branca=1) \\
estudo3 & Sem instrução ou com menos de 1 ano freqüentado \\
estudo4 & Com pelo menos 4 anos de escolaridade \\
rural & Com pelo menos 8 anos de escolaridade \\
Metro & Com pelo menos 11 anos de escolaridade \\
\hline
\end{tabular}

Fonte: Elaborado na pesquisa.

2 O questionário com suplemento sobre programas sociais foi aplicado pela PNAD 2004, todavia não foi utilizado neste estudo. 
Foram selecionadas apenas pessoas entre 15 e 65 anos, ou seja, indivíduos que se beneficiam das transferências monetárias do PBF, uma vez que elas próprias podem ser elegíveis ou compartilhem o domicílio com crianças ou adolescentes que sejam.

As variáveis que determinam a participação no PBF são as características domiciliares. Neste sentido, elencou-se um conjunto de variáveis sociais, de localização geográfica e de características observáveis dos indivíduos. Os impactos do PBF sobre as variáveis foram investigados a partir de dois recortes iniciais e quatro recortes secundários: o primeiro foi composto por homens e mulheres no Brasil; e o seguinte foi estruturado por mulheres e homens chefe e mulheres e homens cônjuges. A descrição das covariáveis que compõe o modelo que mensura a probabilidade de participação de seleção do programa (escore de propensão) estão na Tabela 1 acima.

\section{Método de Análise de Causalidade}

Inicialmente, para identificar o efeito do benefício do PBF sobre a participação no mercado de trabalho é estimado um modelo lógite. Desse modo, partindo do ponto central desse trabalho, que é o de discutir sobre a relação causa-efeito entre o recebimento de transferências de renda e a participação na oferta de trabalho dos indivíduos, faz-se necessário um maior aprofundamento empírico a respeito da análise de causalidade. Os fatores envolvidos na alteração das horas trabalhadas podem ser diversos, todavia cabe a identificação da forma como o recebimento do PBF está atrelado à essas alterações e não aos demais fatores.

De acordo com Brady (2003) para estabelecer a causalidade, apenas a identificação entre causa e efeito não bastam. A direção da relação causal se torna imprescindível, assim como a unicidade da causa. Para discutir a respeito desta, a Teoria Contrafactual é a que mais se adéqua ao desenho do PBF. De acordo com Brady (2008), esta teoria baseia-se no pressuposto de que quando ocorre a causa, o efeito também ocorre, caso contrário ambas situações não serão percebidas ${ }^{3}$. A causalidade está assegurada sob a hipótese de que as duas situações sejam idênticas.

Desse modo pode-se compreender que o impacto será a diferença observada na variável de interesse quando a causa estiver presente e quando ela não estiver. Seja $T_{n}(1)$ a participação no mercado de trabalho na presença da causa, ou seja o choque orçamentário proveniente de transferências de renda do PBF, e $T_{n}(0)$ a participação no mercado de trabalho na ausência das transferências, tem-se o seguinte impacto:

3 De acordo com Brady (2008), para o cumprimento da causalidade tem-se: a) quando A ocorre, B ocorre; e b) quando A não ocorre, B não ocorre. 


$$
\Delta T_{n}=T_{n}(1)-T_{n}(0)
$$

Tratando-se de um experimento, quando os indivíduos são expostos à uma distribuição aleatória da causa há garantia que, na média, os grupos expostos à causa sejam equivalentes ao grupo dos que não foram. Neste caso, a diferença entre a variável resposta média estimada para o grupo tratamento $\left(T_{n t}\right)$ e a resposta média para o grupo comparação $\left(T_{n c}\right)$ nos dá o Efeito Médio do Tratamento $^{4}(A T E)$.

$$
A T E=E\left[T_{n t}-T_{n c}\right]
$$

Ao restringir a análise ao grupo de tratados, o resultado passa a designar-se Efeito Médio do Tratamento sobre os Tratados (ATT). Dessa forma, sendo $t$ uma binária que indica sobre a participação no programa, tem-se:

$$
A T T=t\left[T_{n t}-T_{n c}\right]
$$

O impacto do programa pode ser comprometido caso as características dos grupos não sejam idênticas na média, o que pode influenciar no efeito gerando um viés na mensuração. Dessa forma, há uma atenção especial para pontos que envolvem as características individuais determinantes na participação no mercado de trabalho, sendo que o PBF seria apenas uma das causas para o possível efeito na participação no mercado de trabalho, sendo necessário um tratamento adequado das causas nos dados disponíveis.

De acordo com Przerwoski (2007) a garantia da identificação da causa no contexto de quase-experimentos é dada por duas condições, a saber: a homogeneidade e a independência média condicionada. A primeira alega que dois domicílios terão o mesmo comportamento frente à causa caso eles tenham as mesmas características observáveis e não observáveis. Já a segunda pressupõe que as características não observáveis não são fonte de viés, ou seja, são irrelevantes na identificação da causa.

Przerwoski (2007) afirma que a variável resposta é a soma dos resultados para os grupos de tratados e controle, assim:

$$
T_{n}=t T_{n}(1)+(1-t) T_{n}(0)
$$


Sendo:

$t$ : presença do tratamento $t=1$;

$T_{n}(t)$ : variável resposta horas de trabalho;

O efeito médio condicionado às covariadas pode ser representado da seguinte forma:

$$
E\left[T_{n} \mid H, R\right]=E\left[T_{n} \mid H\right]+U
$$

Sendo que, $H$ : Vetor de covariadas observáveis determinantes do recebimento do tratamento; $R$ : Vetor de covariadas não observáveis; $U$ : efeito de em $Y$.

Substituindo (5) em (4), tem-se:

$$
\begin{aligned}
& T_{n}=t\left(E\left[T_{n}(1) \mid H\right]+U(1)\right)+(1-t)\left(E\left[T_{n}(0) \mid H\right]+U(0)\right) \\
& T_{n}=E\left[T_{n}(0) \mid H\right]+U(0)+t\left(E\left[T_{n}(1) \mid H\right]-E\left[T_{n}(0) \mid H\right]+U(1)-U(0)\right) \\
& T_{n}=E\left[T_{n}(0) \mid H\right]+t\left(E\left[T_{n}(1)-T_{n}(0) \mid H\right]\right)+t U(1)-t U(0)+U(0) \\
& T_{n}=E\left[T_{n}(0) \mid H\right]+t\left(E\left[T_{n}(1)-T_{n}(0) \mid H\right]\right)+U
\end{aligned}
$$

A participação no mercado de trabalho é calculada encontrando a média dos grupos de tratamento e controle, quando o choque orçamentário não estiver presente, somada à alteração desencadeada na participação no mercado de trabalho do grupo que foi afetado pela causa e aos fatores não observáveis. $\mathrm{O}$ impacto do tratamento $\Delta T_{n}$ nos tratados é então, representado da seguinte forma:

$$
A T T=t E\left[T_{n}(1)-T_{n}(0) \mid H\right]
$$

Para que as variáveis não observáveis não estejam correlacionadas com o efeito, é necessário que as condições de homogeneidade, independência média condicionada e $E(U)=0$ sejam satisfeitas, validando assim a expressão acima. 
A suposição de independência dos grupos tratado e controle se faz necessário a medida que se passa a acreditar que desta maneira o resultado obtido não seja subestimado (ou superestimado no caso de o grupo de controle reagir no sentido contrário ao tratamento), evitando assim o viés decorrente do contato dos grupos.

Dentre os métodos amplamente usados para análise empírica embasados na Teoria Contrafactual, pode-se destacar o Escore de Propensão e o Pareamento por Escore de Propensão. Ambos são apresentados na sessão seguinte.

\section{Pareamento pelo Escore de Propensão}

A necessidade de um método que viabilizasse o pareamento com um número mais elevado de variáveis fez com que o conceito de Escore de Propensão fosse desenvolvido. Bryson et al. (2002) faz alusão ao método de pareamento dentro da amostra, em que domicílios mais semelhantes o possível dos que receberam o benefício são selecionados, sendo comparados posteriormente, dois a dois, de acordo com as características observáveis. Pares suficientemente semelhantes devem ser encontrados, mesmo que haja domicílios heterogêneos entre os grupos de controle e tratamento.

O pareamento é um procedimento não paramétrico que corresponde ao condicionamento de efeito nas covariadas observáveis. De acordo com a Teoria Contrafactual, para que sejam satisfeitas as condições para identificação da causa, $\left[D(t) \perp T_{n}(t) \mid H\right]$, as condições de homogeneidade, independência média condicionada e não correlações das variáveis não observáveis com o efeito devem ser satisfeitas. Desse modo, a distribuição do tratamento $D(t)$ determinado pelas características observáveis é o pressuposto essencial para identificação da causa.

De acordo com Dehejia e Wahba (2002) a existência de critérios ajuda a garantir um pareamento adequado. $\mathrm{O}$ critério do suporte comum dos tratados e controle é um desses, pois colabora para que haja um percentual suficiente de domicílios em ambos os grupos, contendo cada uma das características relevantes. Já o outro critério é o de balanceamento que utiliza testes para estabelecer se o grupo de controle tem na média as mesmas probabilidades de ser beneficiado que o grupo tratado. Os testes são feitos tentando assegurar a ortogonalidade entre as covariadas e a variável dependente, já que se corrige para o Escore de Propensão.

De acordo com Imbens (2000), sendo $D(t)$ a distribuição do tratamento; $t$ um indicador de domicílios que recebem o benefício, em que $t=0$ corresponde à não beneficiários e $t=1$ são os beneficiários do programa; $T_{n}(t)$ a variável 
resposta, participação no mercado de trabalho, em função de receber ou não o PBF; e $P(H)=\operatorname{pr}(t=1 \mid H=h)$ a probabilidade de receber o tratamento condicional às covariadas observáveis determinantes da distribuição do tratamento relacionadas ao domicílio.

Portanto, o efeito do tratamento na variável de resultado, que é a participação laboral, é obtido da seguinte forma:

$$
A T T=t E\left[T_{n}(1)-T_{n}(0) \mid P(H)\right]
$$

De acordo com Becker e Ichino (2002), as metodologias que mais se destacam na literatura de avaliação são: Pareamento pelo Vizinho Mais Próximo (Nearest Neighbor Matching); Pareamento pelo raio (Radius matching); Pareamento Estratificado (Stratification matching); e o Pareamento de Kernel (Kernel Matching).

As diferentes soluções propostas para o trade offentre qualidade e quantidade dos pareamentos, propostos pelas metodologias citadas, leva à conclusão de que não há hierarquia entre elas. Logo, a utilização conjunta dessas metodologias oferece uma ferramenta importante para comparar a robustez das estimativas.

Optou-se pela utilização do Método de Kernel ${ }^{5}$. A escolha desse estimador não paramétrico deu-se devido à sua metodologia, que calcula a diferença de média entre tratados e controle ponderando pelo escore de propensão. Dessa forma, famílias do grupo de controle que são muito semelhantes a famílias do grupo de tratamento, ou seja, que apresentem elevado escore de propensão recebem peso maior no momento da estimação do efeito do tratamento, uma vez que representam melhor as famílias do tratamento. Além disso, o fato de empregar todas as observações da amostra faz com que sua variância seja menor que a dos outros estimadores (Caliendo; Kopeinig, 2008).

\section{Evidências do efeito do Programa Bolsa Família na participação de homens e mulheres no mercado de trabalho}

\subsection{Análise do modelo lógite}

A partir da seleção das diversas variáveis relacionadas aos recursos familiares, realizaram-se testes para se obter o modelo mais adequado para o procedi- 
mento de pareamento pelo escore de propensão. Este estudo baseia-se em um modelo lógite, com dois desdobramentos, que foram obtidos a partir de grupos distintos: mulheres chefe no domicílio e mulheres cônjuges; e homens de referência e homens cônjuges. Os resultados dos modelos lógite apontaram que a maioria das variáveis são estatisticamente significativas.

A especificação final do modelo lógite do escore de propensão, observado na tabela 2, satisfez a hipótese do balanceamento (Balancing Hypothesis) das variáveis específicas incluídas, ou seja, as médias de todas as variáveis não apresentaram diferença significativa entre os grupos de tratamento e controle, além disso, os resultados dos coeficientes estimados pelo modelo para grande parte das variáveis apresentaram os sinais esperados.

Em relação às mulheres chefes no Brasil, ao nível de significância de 1\% os dados apontam relação positiva para as variáveis idade, estudo1, estudo2, estudo3, e nbranco com o recebimento do PBF. Dessa forma, domicílios compostos por mulheres chefes que participam do PBF e apresentam membros com idade mais elevada (comparado com os homens chefes), com nível de escolaridade de até oito anos, não brancos e habitam regiões metropolitanas, prevalecem como características principais.

Por outro lado, os dados acusaram relação negativa entre as mulheres chefes que recebem recursos do PBF e habitação em áreas rurais, ao nível de significância de $5 \%$ para o Brasil. As regiões metropolitanas também apresentam relação negativa em todas as posições domiciliares analisadas, obtendo um nível de significância de $1 \%$. 
TABELA 2: ANÁLISE LÓGITE PARA MULHERES E HOMENS, BRASIL E PARANÁ

\begin{tabular}{|c|c|c|c|c|c|c|c|}
\hline \multirow[b]{2}{*}{$\begin{array}{l}\text { Variá- } \\
\text { vel }\end{array}$} & \multicolumn{3}{|c|}{ BRASIL } & \multicolumn{4}{|c|}{ PARANÁ } \\
\hline & $\begin{array}{c}\text { Mu- } \\
\text { lheres } \\
\text { Chefe }\end{array}$ & $\begin{array}{c}\text { Mu- } \\
\text { lheres } \\
\text { Cônju- } \\
\text { ges } \\
\end{array}$ & $\begin{array}{c}\text { Ho- } \\
\text { mens } \\
\text { Chefe }\end{array}$ & $\begin{array}{c}\text { Ho- } \\
\text { mens } \\
\text { Côn- } \\
\text { juge }\end{array}$ & $\begin{array}{l}\text { Mu- } \\
\text { lheres } \\
\text { Chefe }\end{array}$ & $\begin{array}{c}\text { Mu- } \\
\text { lheres } \\
\text { Cônju- } \\
\text { ge } \\
\end{array}$ & $\begin{array}{c}\text { Ho- } \\
\text { mens } \\
\text { Chefe }\end{array}$ \\
\hline \multirow{2}{*}{ Idade } & $0,399^{*}$ & $0,402^{*}$ & $0,209^{*}$ & $-0,016$ & $0,466^{*}$ & $0,425^{*}$ & $0,331^{*}$ \\
\hline & $(0,011)$ & $(0,008)$ & $(0,02)$ & 0,055 & 0,088 & 0,064 & 0,128 \\
\hline \multirow{2}{*}{ idade2 } & $-0,005^{*}$ & $-0,005^{*}$ & $-0,002^{*}$ & 0,001 & $-0,006^{*}$ & $-0,006^{*}$ & $-0,004 *$ \\
\hline & 0,000 & 0,000 & 0,000 & 0,001 & 0,001 & 0,001 & 0,002 \\
\hline \multirow{2}{*}{ Nbranco } & $0,284^{*}$ & $0,328^{*}$ & $-0,017$ & 0,236 & 0,020 & 0,268 & 0,186 \\
\hline & 0,046 & 0,031 & 0,080 & 0,284 & 0,284 & 0,179 & 0,381 \\
\hline \multirow{2}{*}{ estudo1 } & $1,638^{*}$ & $1,300^{*}$ & 1,241 & $1,370^{*}$ & $1,760^{*}$ & 0,990 & $1,603^{*}$ \\
\hline & 0,333 & 0,211 & 1,008 & 0,994 & 1,605 & 1,082 & 1,120 \\
\hline \multirow{2}{*}{ estudo2 } & $1,617^{*}$ & $1,272^{*}$ & 1,499 & $1,383^{*}$ & $1,748^{*}$ & 1,019 & $1,564^{*}$ \\
\hline & 0,33 & 0,21 & 1,005 & 0,932 & 1,599 & 1,067 & 1,060 \\
\hline \multirow{2}{*}{ estudo3 } & $1,469^{*}$ & $0,992^{*}$ & 1,222 & $1,393^{*}$ & $1,705^{*}$ & 0,990 & $1,578^{*}$ \\
\hline & 0,330 & 0,210 & 1,006 & 0,877 & 1,569 & 1,082 & 1,062 \\
\hline \multirow{2}{*}{ estudo4 } & $0,829^{* *}$ & $0,421^{* *}$ & 0,850 & $1,373^{*}$ & $1,636^{*}$ & 1,019 & $1,429 *$ \\
\hline & 0,331 & 0,211 & 1,011 & 0,915 & 1,552 & 1,067 & 1,552 \\
\hline \multirow{2}{*}{ Rural } & $-0,009$ & $0,454^{*}$ & $0,152^{* *}$ & 0,019 & $-0,159$ & 0,585 & 0,464 \\
\hline & 0,058 & o,o31 & 0,081 & 0,287 & 0,418 & 1,069 & 0,388 \\
\hline \multirow{2}{*}{ Metro } & $-0,444^{*}$ & $-0,618^{*}$ & $-0,426^{*}$ & $-0,221$ & $-0,129$ & $0,318 * *$ & $-0,369$ \\
\hline & 0,044 & 0,036 & 0,100 & 0,277 & 0,281 & 1,082 & 0,472 \\
\hline \multirow{2}{*}{ _cons } & $-1,306^{*}$ & $-9,404^{*}$ & $-9,317^{*}$ & $-19,381$ & $-26,898$ & $-9,126^{*}$ & $-26,07^{*}$ \\
\hline & 0,386 & 0,257 & 1,074 & - & - & 1,516 & 2,702 \\
\hline
\end{tabular}

Legenda: * Significativo em nível de 1\%; ** Significativo em nível de 5\%.

Fonte: Elaborado com base na PNAD 2006.

Analisando o modelo para mulheres cônjuges no Brasil que recebem bolsa, observa-se a relação positiva entre as variáveis idade, estudo1, estudo2, estudo3 e rural com 1\% de significância. Em relação aos homens chefes beneficiários do PBF no Brasil, apenas a variável idade e metro demonstraram-se significativas à $1 \%$. A habitação em áreas rurais teve um nível de significância de $5 \%$. As demais variáveis não foram significativas. Já para os homens cônjuges as variáveis estudo1, estudo2, estudo3, estudo4 apresentaram-se com $1 \%$ de significância.

Na análise para o Paraná os níveis de escolaridade demonstraram-se significativos a $1 \%$ tanto para as mulheres chefes quanto para os homens. No caso das mulheres cônjuges apenas as variáveis idade e metro foram significativas a 1\% e 5\%, respectivamente. A análise para os homens não pode ser concluída devido à restrição da amostra. 


\subsection{Estabelecendo relações: o PBF e o mercado de traba- \\ lho}

Afim de obter melhores resultados, ampliou-se a especificação da análise realizada neste trabalho. Decidiu-se criar desdobramentos a partir da posição domiciliar ocupadas por homens e mulheres, tanto no Brasil quanto no Paraná. A posição domiciliar declarada à PNAD 2006 e utilizada nas análises são as dos chefes e cônjuges.

Focalizando nas mulheres chefes, os resultados mostram que a proporção das que participam do mercado de trabalho e que são beneficiárias do PBF apresentou-se significativo para o Método de Kernel e o Método do raio= o,o01. Pode-se ver que o PBF acarreta um aumento na participação do mercado de trabalho de 2,3 pontos percentuais (Tabela 5) das mulheres chefes do domicílio, sendo que foi considerado apenas o Método de Kernel por motivos já especificados anteriormente. Em relação aos homens chefes, nota-se que o PBF elevou mais intensamente a proporção no mercado de trabalho comparando-se com as mulheres. Os 4,8 pontos percentuais (Tabela 5) representam a relação de homens beneficiários do programa que participam do mercado de trabalho.

Analisando apenas o gênero dos beneficiários no Brasil, vê-se pela tabela 8 que tanto as mulheres quanto os homens apresentaram um ATT significativo, todavia o nível de significância apresentado pelas mulheres foi bem mais elevado. Desse modo, as mulheres beneficiárias elevam sua participação no mercado de trabalho em 1,8 pontos percentuais, já os homens elevam em 4,6 pontos percentuais. 
TABELA 3: ESTIMADOR DO TESTE DE DIFERENÇAS ENTRE MÉDIAS (ATT), BRASIL

\begin{tabular}{|c|c|c|c|c|c|}
\hline \multirow[b]{2}{*}{ Método } & \multicolumn{5}{|c|}{ Mulheres } \\
\hline & $\begin{array}{l}\text { Número } \\
\text { tratados }\end{array}$ & $\begin{array}{l}\text { Número } \\
\text { controle }\end{array}$ & ATT & $\begin{array}{c}\text { Erro } \\
\text { padrão }\end{array}$ & $\mathbf{t}$ \\
\hline Kernel & 11248 & 34715 & 0,018 & 0,001 & 13,019 \\
\hline Estratificação & 11248 & 34715 & 0,005 & 0,005 & 0,999 \\
\hline Raio=0,001 & 11248 & 34689 & 0,023 & 0,006 & 3,600 \\
\hline Raio $=0,0001$ & 11210 & 31595 & $-0,008$ & 0,007 & $-1,199$ \\
\hline Raio $=0,00001$ & 11140 & 30245 & $-0,029$ & 0,006 & $-5,081$ \\
\hline $\begin{array}{l}\text { Vizinho mais } \\
\text { Próximo }\end{array}$ & 11248 & 30197 & 0,001 & 0,001 & 0,953 \\
\hline \multirow[b]{2}{*}{ Método } & \multicolumn{5}{|c|}{ Homens } \\
\hline & $\begin{array}{l}\text { Número } \\
\text { tratados }\end{array}$ & $\begin{array}{l}\text { Número } \\
\text { controle }\end{array}$ & ATT & $\begin{array}{c}\text { Erro } \\
\text { padrão }\end{array}$ & $\mathbf{t}$ \\
\hline Kernel & 797 & 40071 & 0,046 & 0,010 & 4,494 \\
\hline Estratificação & 797 & 40071 & 0,007 & 0,011 & 0,647 \\
\hline Raio=0,001 & 797 & 39876 & o,o13 & 0,004 & 1,765 \\
\hline Raio $=0,0001$ & 718 & 36987 & $-0,005$ & 0,003 & $-1,045$ \\
\hline Raio $=0,00001$ & 679 & 34562 & $-0,027$ & 0,007 & $-7,043$ \\
\hline $\begin{array}{l}\text { Vizinho mais } \\
\text { Próximo }\end{array}$ & 797 & 18594 & $-0,003$ & 0,004 & $-0,645$ \\
\hline
\end{tabular}

Fonte: Elaborado com base na PNAD 2006.

Tratando-se do Paraná, conclui-se que as diferenças nas médias de participação no mercado de trabalho entre as mulheres cônjuges segundo participação no PBF são estatisticamente significativas, como observado na tabela 8 , com elevação da participação no mercado de trabalho em 3,1 pontos percentuais. No caso dos homens os valores não são significativos, o que sugere que não há efeitos na participação no mercado de trabalho por parte dos beneficiários na atividade laboral do Estado. 
TABELA 4: ESTIMADOR DO TESTE DE DIFERENÇAS ENTRE MÉDIAS (ATT), PARANÁ

\begin{tabular}{|c|c|c|c|c|c|}
\hline \multirow[b]{2}{*}{ Método } & \multicolumn{5}{|c|}{ Mulheres } \\
\hline & $\begin{array}{l}\text { Número } \\
\text { tratados }\end{array}$ & $\begin{array}{l}\text { Número } \\
\text { controle }\end{array}$ & ATT & $\begin{array}{c}\text { Erro } \\
\text { padrão }\end{array}$ & $\mathbf{t}$ \\
\hline Kernel & 248 & 1182 & 0,031 & 0,010 & 2,978 \\
\hline Estratificação & 248 & 1182 & 0,026 & 0,034 & 0,761 \\
\hline Raio $=0,001$ & 239 & 703 & 0,013 & 0,036 & 0,365 \\
\hline Raio $=0,0001$ & 172 & 259 & $-0,060$ & 0,058 & $-1,042$ \\
\hline Raio=0,00001 & 129 & 166 & $-0,039$ & 0,045 & $-0,863$ \\
\hline $\begin{array}{l}\text { Vizinho mais } \\
\text { Próximo }\end{array}$ & 248 & 274 & $-0,032$ & 0,036 & $-0,885$ \\
\hline \multirow[b]{2}{*}{ Método } & \multicolumn{5}{|c|}{ Homens } \\
\hline & $\begin{array}{l}\text { Número } \\
\text { tratados }\end{array}$ & $\begin{array}{l}\text { Número } \\
\text { controle }\end{array}$ & ATT & $\begin{array}{c}\text { Erro } \\
\text { padrão }\end{array}$ & $\mathbf{t}$ \\
\hline Kernel & 30 & 1006 & $-0,009$ & 0,043 & $-0,203$ \\
\hline Estratificação & 30 & 1006 & $-0,006$ & 0,064 & $-0,093$ \\
\hline Raio $=0,001$ & 30 & 599 & $-0,024$ & 0,056 & $-0,440$ \\
\hline Raio $=0,0001$ & 24 & 107 & 0,056 & 0,080 & 0,699 \\
\hline Raio=0,0000 1 & 18 & 38 & 0,023 & 0,062 & 0,379 \\
\hline $\begin{array}{l}\text { Vizinho mais } \\
\text { Próximo }\end{array}$ & 73 & 1933 & 0,014 & 0,016 & 0,853 \\
\hline
\end{tabular}

Fonte: Elaborado com base na PNAD 2006.

A significância para o método principal evidenciado, no caso das mulheres e homens chefes, pode ser explicado a partir da participação do cônjuge nos domicílios entrevistados, que no caso dos homens demonstrou-se bem menos significativo, como se pode observar nas tabelas 5 e 6 . Na maioria dos grupos domésticos, a postura da mãe em relação à saúde, educação e tudo que se trate dos filhos é mais presente do que a dos homens, uma vez que as atividades domésticas são praticadas majoritariamente pelas mulheres. Desse modo, as mulheres permanecem um número de horas maior nos domicílios quando comparado com os homens e isso pode explicar o motivo pela qual a participação das mulheres apresentou um valor pouco mais baixo do que os homens chefes quando se trata de participação do mercado de trabalho brasileiro. 
TABELA 5: EFEITO MÉDIO DO TRATAMENTO SOBRE OS TRATADOS (ATT), BRASIL

\begin{tabular}{|c|c|c|c|c|c|}
\hline \multirow[b]{2}{*}{ Método } & \multicolumn{5}{|c|}{ Mulheres chefes } \\
\hline & $\begin{array}{l}\text { Número } \\
\text { tratados }\end{array}$ & $\begin{array}{l}\text { Número } \\
\text { controle }\end{array}$ & ATT & $\begin{array}{c}\text { Erro } \\
\text { padrão }\end{array}$ & $\mathbf{t}$ \\
\hline Kernel & 3327 & 18383 & 0,023 & 0,003 & 6,935 \\
\hline Estratificação & 3327 & 18383 & 0,006 & 0,010 & 0,611 \\
\hline Raio $=0,001$ & 3327 & 18270 & 0,029 & 0,011 & 2,574 \\
\hline Raio $=0,0001$ & 2683 & 14771 & $-0,040$ & 0,015 & $-2,701$ \\
\hline Raio=0,00001 & 1983 & 13176 & $-0,070$ & 0,013 & $-5,465$ \\
\hline $\begin{array}{l}\text { Vizinho mais } \\
\text { Próximo }\end{array}$ & 3357 & 12935 & 0,002 & 0,004 & 0,405 \\
\hline \multirow[b]{2}{*}{ Método } & \multicolumn{5}{|c|}{ Homens chefes } \\
\hline & $\begin{array}{l}\text { Número } \\
\text { tratados }\end{array}$ & $\begin{array}{l}\text { Número } \\
\text { controle }\end{array}$ & ATT & $\begin{array}{c}\text { Erro } \\
\text { padrão }\end{array}$ & $\mathbf{t}$ \\
\hline Kernel & 774 & 37756 & 0,048 & 0,011 & 4,222 \\
\hline Estratificação & 774 & 37756 & 0,007 & 0,011 & 0,654 \\
\hline Raio $=0,001$ & 774 & 37756 & 0,020 & 0,009 & 2,328 \\
\hline Raio $=0,0001$ & 774 & 33100 & 0,012 & 0,011 & 1,064 \\
\hline Raio=0,00001 & 772 & 20276 & $-0,032$ & 0,014 & $-2,322$ \\
\hline $\begin{array}{l}\text { Vizinho mais } \\
\text { Próximo }\end{array}$ & 774 & 17192 & o,003 & 0,004 & 0,625 \\
\hline
\end{tabular}

Fonte: Elaborado com base na PNAD 2006.

Os resultados significativos também podem ser atribuídos às condicionalidades, uma vez que a exigência de frequência escolar para que as famílias recebam os benefícios libera horas com atividades de cuidado com os filhos, permitindo a participação mais ativa no mercado de trabalho, especialmente das mulheres.

Também se pode apontar a ocorrência de um efeito substituição, já que muitas das crianças exercem trabalho remunerado desde cedo para complementar a renda familiar. Com a redução da renda proveniente do trabalho infantil, homens e mulheres chefes vêem a necessidade de elevar as horas de trabalho, para compensar ou fornecer um acréscimo ao benefício recebido.

Em relação às mulheres e homens cônjuges, a Tabela 6 revela que apenas para as mulheres cônjuges o efeito foi significativo. Há uma elevação de 2,5 pontos percentuais na participação no mercado de trabalho das beneficiarias do programa. Esse resultado pode ser explicado devido ao fato da maioria dos 
domicílios apresentar uma estrutura familiar monoparental e com pessoas além da estrutura nuclear preferencial. Na maioria dos domicílios o cônjuge (marido ou companheiro) está ausente, o que torna a composição dos grupos domésticos e o cumprimento das condicionalidades do PBF, tarefa das mulheres, diante à ausência de outro representante da família.

TABELA 6: EFEITO MÉDIO DO TRATAMENTO SOBRE OS TRATADOS (ATT), BRASIL

\begin{tabular}{|c|c|c|c|c|c|}
\hline \multirow[b]{2}{*}{ Método } & \multicolumn{5}{|c|}{ Mulheres cônjuges } \\
\hline & $\begin{array}{l}\text { Número } \\
\text { tratados }\end{array}$ & $\begin{array}{l}\text { Número } \\
\text { controle }\end{array}$ & ATT & $\begin{array}{c}\text { Erro } \\
\text { padrão }\end{array}$ & $\mathbf{t}$ \\
\hline Kernel & 8172 & 25543 & 0,025 & 0,002 & 14,349 \\
\hline Estratificação & 8172 & 25543 & 0,010 & 0,006 & 1,705 \\
\hline Raio $=0,001$ & 8172 & 25480 & 0,050 & 0,007 & 7,674 \\
\hline Raio $=0,0001$ & 8127 & 22410 & 0,022 & 0,009 & 2,610 \\
\hline Raio=0,00001 & 8071 & 21061 & 0,013 & 0,007 & 1,843 \\
\hline $\begin{array}{l}\text { Vizinho mais } \\
\text { Próximo }\end{array}$ & 8172 & 20939 & 0,002 & 0,002 & 1,062 \\
\hline \multirow[b]{2}{*}{ Método } & \multicolumn{5}{|c|}{ Homens cônjuges } \\
\hline & $\begin{array}{l}\text { Número } \\
\text { tratados }\end{array}$ & $\begin{array}{l}\text { Número } \\
\text { controle }\end{array}$ & ATT & $\begin{array}{c}\text { Erro } \\
\text { padrão }\end{array}$ & $\mathbf{t}$ \\
\hline Kernel & 73 & 15272 & $-0,076$ & 0,057 & $-1,327$ \\
\hline Estratificação & 73 & 15272 & $-0,046$ & 0,059 & $-0,790$ \\
\hline Raio $=0,001$ & 72 & 15102 & $-0,097$ & 0,075 & $-1,305$ \\
\hline Raio $=0,0001$ & 72 & 13492 & $-0,077$ & 0,075 & $-1,032$ \\
\hline Raio=0,0000 1 & 72 & 4781 & 0,077 & 0,072 & 1,071 \\
\hline $\begin{array}{l}\text { Vizinho mais } \\
\text { Próximo }\end{array}$ & 73 & 1933 & 0,014 & 0,016 & 0,853 \\
\hline
\end{tabular}

Fonte: Elaborado com base na PNAD 2006.

Em suma, os impactos do programa sobre os homens cônjuges se revelaram não significativos, em contraponto no caso das mulheres o efeito mais expressivo deu-se justamente para a posição de cônjuge, já que a maioria das mulheres ocupa essa posição nos domicílios. A posição de pessoa de referência para ambos os sexos foi significante, sendo mais representativo no caso das mulheres. Em geral, os efeitos do programa sobre a participação no mercado de trabalho foram altamente positivos para ambos os sexos, especialmente para os chefes dos domicílios. 
TABELA 7: EFEITO MÉDIO DO TRATAMENTO SOBRE OS TRATADOS (ATT), PARANÁ

\begin{tabular}{|c|c|c|c|c|c|}
\hline \multirow[b]{2}{*}{ Método } & \multicolumn{5}{|c|}{ Mulheres chefes } \\
\hline & $\begin{array}{l}\text { Número } \\
\text { tratados }\end{array}$ & $\begin{array}{l}\text { Número } \\
\text { controle }\end{array}$ & ATT & $\begin{array}{c}\text { Erro } \\
\text { padrão }\end{array}$ & $\mathbf{t}$ \\
\hline Kernel & 69 & 390 & 0,018 & 0,013 & 1,387 \\
\hline Estratificação & 68 & 391 & 0,021 & 0,056 & 0,368 \\
\hline Raio $=0,001$ & 55 & 114 & 0,091 & 0,093 & 0,982 \\
\hline Raio $=0,0001$ & 25 & 36 & $-0,040$ & 0,120 & $-0,333$ \\
\hline Raio $=0,00001$ & 21 & 27 & $-0,094$ & 0,086 & $-1,090$ \\
\hline $\begin{array}{l}\text { Vizinho mais } \\
\text { Próximo }\end{array}$ & 69 & 69 & 0,043 & 0,062 & 0,705 \\
\hline \multirow[b]{2}{*}{ Método } & \multicolumn{5}{|c|}{ Homens chefes } \\
\hline & $\begin{array}{l}\text { Número } \\
\text { tratados }\end{array}$ & $\begin{array}{l}\text { Número } \\
\text { controle }\end{array}$ & ATT & $\begin{array}{c}\text { Erro } \\
\text { padrão }\end{array}$ & $\mathbf{t}$ \\
\hline Kernel & 30 & 954 & $-0,009$ & 0,040 & $-0,213$ \\
\hline Estratificação & 30 & 954 & $-0,011$ & 0,058 & $-0,193$ \\
\hline Raio $=0,001$ & 30 & 531 & $-0,022$ & 0,048 & $-0,465$ \\
\hline Raio $=0,0001$ & 24 & 124 & o,030 & 0,074 & 0,400 \\
\hline Raio=0,00001 & 16 & 35 & $-0,005$ & 0,066 & $-0,082$ \\
\hline $\begin{array}{l}\text { Vizinho mais } \\
\text { Próximo }\end{array}$ & 30 & 51 & $-0,033$ & 0,076 & $-0,439$ \\
\hline
\end{tabular}

Fonte: Elaborado com base na PNAD 2006.

Analisando o Estado do Paraná, as mulheres e homens chefes (Tabela 7) obtiveram um ATT não significativo. Apenas as mulheres cônjuges do Estado apresentaram valores do Método de Kernel significativo, com um ATT que representa uma elevação de 3,1 pontos percentuais na participação no mercado de trabalho.

Para os homens cônjuges no Paraná os valores dos métodos de pareamento não puderam ser obtidos devido a restrição nos números da amostra. 
TABELA 8: EFEITO MÉDIO DO TRATAMENTO SOBRE OS TRATADOS (ATT), MULHERES CÔNJUGES, PARANÁ

\begin{tabular}{lccccc}
\hline Método & $\begin{array}{c}\text { Número } \\
\text { tratados }\end{array}$ & $\begin{array}{c}\text { Número } \\
\text { controle }\end{array}$ & ATT & $\begin{array}{c}\text { Erro } \\
\text { padrão }\end{array}$ & t \\
\hline Kernel & 180 & 911 & 0,031 & 0,015 & 2,086 \\
Estratificação & 180 & 911 & 0,012 & 0,043 & 0,273 \\
Raio=0,001 & 174 & 446 & $-0,014$ & 0,050 & $-0,287$ \\
Raio=0,0001 & 107 & 152 & 0,039 & 0,068 & 0,574 \\
Raio=0,00001 & 86 & 97 & $-0,063$ & 0,053 & $-1,202$ \\
Vizinho mais & 180 & 185 & $-0,011$ & 0,050 & $-0,222$ \\
Próximo & & & & & \\
\hline
\end{tabular}

Fonte: Elaborado com base na PNAD 2006.

Desse modo, pode-se afirmar a importância do programa tanto no âmbito nacional quanto no regional. A análise para as mulheres apresentou-se mais significante tanto para o Brasil quanto para o Paraná. À medida que as posições domiciliares foram sendo incluídas, comprovou-se que apenas para as mulheres chefes no Paraná, que recebem o PBF, a participação não foi alterada com a presença dos recursos.

Estendendo a análise para os homens, o fato de ser cônjuge dentro da posição domiciliar, faz com que a participação no mercado de trabalho não seja alterada com recebimento do $\mathrm{PBF}$, isso para o Brasil. Já para o Paraná a amostra não foi suficiente para realizar os métodos necessários, devido ao número de homens que se declaram cônjuges no Estado.

Contatou-se então que o PBF pode, na maioria dos casos analisados e principalmente no caso das mulheres, orientar a uma maior participação para o mercado de trabalho. Foi confirmado também que a participação varia de acordo com o sexo e posição do membro no domicílio. Assim, o PBF pode modificar ou reforçar a divisão intradomiciliar do trabalho e, devido ao desenho do programa que favorece as mulheres como beneficiárias oficiais pode criar meios de inserção ao mercado de trabalho e elevar a segurança de renda nos domicílios.

Em geral, os resultados desse trabalho sugerem que o efeito do PBF para a participação no mercado de trabalho dos homens é maior, todavia há mais resultados estatisticamente significativos quando se trata das mulheres. Ao realizar a desagregação, viu-se que o impacto do PBF para mulheres cônjuges comparado com às chefes, tanto no Brasil como no Paraná, também é mais elevado. 


\section{Considerações Finais}

Neste trabalho foram investigados os impactos do PBF sobre a participação no trabalho brasileiro levando-se em conta o sexo e a posição domiciliar dos membros. Assumiu-se o pressuposto que acréscimos da renda provenientes de transferências do governo levariam à desestímulos ao trabalho, acarretado pelo efeito renda e que a retirada de crianças do trabalho infantil e o seu envio à escola pode ter repercutido como uma queda na renda familiar, ocasionando o efeito substituição.

A metodologia utilizada foi a do Pareamento pelo Escore de Propensão que é capaz de gerar, a partir de inúmeros algoritmos, grupos de controle correspondentes com o grupo de tratados para cada família. Estes grupos são capazes de reunir famílias que têm probabilidade de participar do programa semelhante às que já participam. A metodologia tem como ponto fundamental a redução do viés resultante da comparação de famílias com variáveis distintas. Neste estudo, o Pareamento pelo Escore de Propensão analisou os efeitos do PBF sobre a participação de mulheres e homens, diferenciando-os pela posição que ocupam dentro do domicílio.

Para o Brasil, os resultados desse estudo sugerem que o PBF impactou de forma positiva na participação no mercado de trabalho das mulheres, tanto das chefes quanto das cônjuges, por sua vez, para o Paraná, apenas no caso das mulheres cônjuges foram obtidos valores significativos. Notou-se que o PBF contribui também para a participação de homens chefes no Brasil, o que já não ocorre no Paraná. Contudo, para os homens cônjuges no Brasil e os homens chefes no Paraná não foram obtidas estimativas estatisticamente significativas, apontando que não há diferenças relevantes na participação entre os beneficiários e não beneficiários do PBF.

Para o Paraná não foi possível identificar métodos de pareamento para os homens cônjuges devido a restrição na amostra para as características analisadas. Cabe ressaltar, por sua vez, que a maioria dos domicílios declara os homens como pessoas de referência sendo que o número de homens cônjuges é reduzido comparado com o número de mulheres que declaram esta mesma posição no domicílio.

Com a utilização do Método de Kernel se buscou uma maior robustez à análise e, com ele pode-se notar a significância estatística tanto para as mulheres chefes quanto para os homens no Brasil. Com um ATT mais elevado do que o observado nas mulheres chefes, todavia com uma significância mais baixa, os homens chefes que recebem recursos do PBF têm a capacidade de elevar a participação no mercado de trabalho em 4,8 pontos percentuais. Já para as mulheres chefes beneficiárias do programa o efeito na participação é de 2,3 pontos percentuais. Para as mulheres cônjuges o efeito é de 2,5 pontos percentuais com um alto nível de significância. Os homens cônjuges não 
obtiveram valores significativos, não apresentando efeitos na participação no mercado de trabalho.

No Paraná apenas as mulheres cônjuges obtiveram valores significativos, com um ATT equivalente a 3,1 pontos percentuais de elevação na participação laboral das beneficiárias do PBF. Já as mulheres e homens chefes não obtiveram significância estatística e os homens cônjuges não apresentaram amostra suficiente para a análise.

O efeito substituição pode ocorrer nos casos observados, já que como os valores das transferências não são elevados, eles apenas serviriam para incrementar a renda. Como entre as condicionalidades há a obrigatoriedade da frequência de crianças e adolescentes, muitas dessas deixam o trabalho infantil e com ele a renda para a família. A partir daí, pode-se haver uma necessidade maior por parte dos membros de complementar a renda domiciliar perdida com a mão de obra infantil e consequente aumento na participação no mercado de trabalho.

Outro ponto importante para entender o comportamento dos membros beneficiários do PBF diante o mercado de trabalho está na liberação de horas ao enviar as crianças à escola. A partir da diminuição do tempo de cuidado doméstico com os filhos, permite-se a elevação da participação de atividades de trabalho. Como o benefício é dado preferencialmente às mulheres, pode-se notar elevados níveis de significância na análise para mulheres chefes e cônjuges no Brasil e para mulheres cônjuges no Paraná.

Desse modo as perguntas feitas no início deste trabalho foram esclarecidas. Confirmou-se que a participação laboral varia de acordo com o sexo e com o tipo de posição domiciliar do membro. Os resultados mostram que as mulheres são mais sensíveis a oscilações na renda se comparando com os homens, principalmente às do Brasil. As mulheres cônjuges beneficiadas pelo PBF no Paraná são as que se destacam ao se tratar de participação do mercado de trabalho. Logo, o programa pode reforçar a divisão intradomiciliar do trabalho, já que o benefício é dado preferencialmente para as mulheres. Desse modo, é importante considerar dentro da dinâmica do $\mathrm{PBF}$ as posições dos membros, de modo a facilitar a inserção cada vez maior de mulheres no mercado de trabalho, fato que já vem ocorrendo de forma bem intensa, comprovando que a crítica de que o PBF pode gerar dependência dos beneficiários não é pertinente.

\section{Referências}

Barros, R. P.; Foguel, M. N.; Ulyssea, G. Desigualdade de Renda no Brasil: uma análise da queda recente. Brasília: IPEA. 2 v. 2007.

Becker, G. S. The economic approach to human behavior. Chicago: The University of Chicago Press, p. 92, 1976. 
Becker, G. Stanley. A Theory of Allocation of Time. The Economic Journal, Vol. 75, No. 299. p. 493-517. 1965 .

. A treatise on the family. Cambridge: Harvard Univ. 1981.

Becker, S. O. Ichino, A. "Estimation of Average Treatment Effects Based on Propensity Scores," The Stata Journal, 2, 358-377. 2002.

Bichir, R.m. O Bolsa Família na berlinda? Os desafios atuais dos programas de transferência de renda. Revista Novos Estudos - CEBRAP, No 87. São Paulo, julho 2010.

Brady, H, E. Models of causal inference: going beyond the neyman-rubin-holland theory. Chicago: Political Science Association Annual Meeting, 2003.

Brady, Henry. 2008. "Models of Causal Inference: Going Beyond the NeymanRubin-Holland Theory." In Janet M. Box-Steffensmeier, Henry E. Brady, and David Collier, editors, The Oxford Handbook of Political Methodology New York: Oxford University Press.

Brito, Fausto. Transição demográfica e desigualdades sociais no Brasil. Revista Brasileira de Estudos Populacionais, São Paulo, v.25, n.1, p.5-26, jan./jun.2008.

Bryson, Alex; Dorsett, Richard; Purdon, Susan., The use of propensity score matching in the evaluation of active labour market policies. Londres: Department for Work and Pensions, 2002. (Research Working Paper, 4).

Caliendo, M.; Kopeinig, S. Some pratical guidance for the implementation of propensity score matching. IZA Discussion 1588, Institute for the Study of Labor. 2008.

Cameron, A. C.; Trivedi, P. K. Microeconometrics: methods and applications. New York: Cambridge University Press, 2005.

Cardoso, José Celso Jr. Estrutura setorial-ocupacional do emprego no brasil e evolução do perfil distributivo nos anos 9o. Rio de Janeiro: IPEA, 1999. (Working Paper, 655).

Chiappori, P.a. Collective Labor Supply and Welfare. Journal of Political Economy, 100: 437-467, 2002.

Dehejia, Rajeev H.; WAHBA, Sadek. Propensity score-matching methods for nonexperimental causal studies. The Review of Economics and Statistics, Cambridge, v. 84, n. 1, p. 151-161, Feb. 2002.

Duryea, Suzanne, David Lam, and Deborah Levison (2003). Effects of economic shocks on children's employment and schooling in Brazil. PSC report 03-541. Michigan: Population Studies Center, University of Michigan.

Ehrenberg, R.; Smith, R. Modern Labor Economics - Theory and Public Policy. New York: Addison- Wesley.2003.

Ferrario, M.n.; Cunha, M.s. Estrutura e renda familiar no Brasil. Ensaios FEE, Porto Alegre, v.33, n.1, p.123-142, maio 2012.

Ferro, A. R.; Nicollela, A. C. The impact of conditional cash transfer programs on household work decisions in Brazil. Presented at the IZA/World Bank Conference on Employment and Development in 2007. Available at: http://www.iza. org/conference_files/worldb2007/ferro_a3468.pdf $>$.

Foguel, M. N.; Barros, R. P. de. The effects of conditional cash transfer programmes 
on adult labor supply: an empirical analysis using a time-series-cross-section. XXXVI Encontro Nacional de Economia.

Gronau, R. (1977): Leisure, Home Production and Work- The Theory of the Allocation of Time Revisited. Journal of Political Economy, 85: 1099-1124.

Gronau, Reuben. Home production - a survey. In: ASHENFELTER, Orley; LAYARD, P. R. G. Handbook of labor economics. Amsterdam: 1986-1999. v. 1, cap. 4.

Heckman, J. J. Causal parameters and policy analysis in economics: A Twentieth Century Retrospective. Department of Economics, University of Chicago and the American Bar Foundation. June, 1999.

Heckman, J. J.; Ichimura, H.; Todd, P. Matching as an Econometric Evaluation Estimator: Evidence from Evaluating a Job Training Program. Review of Economic Studies. no. 64, p. 605-654. 1997.

Imbens, Guido. The role of the propensity score in estimating dose-response functions, Biometrika, London, v. 87, n. 3, p.706-710, Sep. 2000.

IPEA. Pesquisa Nacional de Amostra de Domicílios (PNAD) 2006: primeiras análises. Brasília: IPEA, 2006.

Parker, S.w.; Skoufias, E. (200o): The Impact of Progresa on Work, Leisure, and Allocation. Final Report, International Food Policy Research Institute, Washington, DC.

Przeworski, Adam. Is the Science of Comparative Politics Possible? In: BOIX, Carles; STOKES, Susan Carol. The Oxford handbook of comparative politics. New York: Oxford University, 2007. (The Oxford handbooks of political science)

Rosenbaum, P.; Rubin, D. B. The Central Role of Propensity Score in Observational Studies for Causal Effects. Biometrika. 70, 41-55. 1983.

Stephens, J. R. M.Worker displacement and the added worker. NBER, Cambridge, 2001. Working Paper, n. 8260.

Teixeira, C. G. Análise da heterogeneidade do Programa Bolsa Família na oferta de trabalho dos homens e das mulheres. Bolsa Família 2003-2010: avanços e desafios/IPEA. Volume 2, capítulo 4. Brasília, 2010. 